

\title{
Estrategias que apoyan el aprendizaje de la caída libre de los cuerpos
}

\section{Strategies that support learning the free fall of bodies}

\author{
BECERRA RODRÍGUEZ, Diego F. ${ }^{1}$ \\ VARGAS SÁNCHEZ, Ana D. ${ }^{2}$ \\ BOUDE FIGUEREDO, Oscar R. ${ }^{3}$ \\ BENÍTEZ MENDIVELSO, Marcela ${ }^{4}$
}

\begin{abstract}
Resumen
Esta investigación analiza la importancia del uso de las TIC y la estrategia de Aprendizaje Activo de la Física, en el aprendizaje de la caída libre de los cuerpos. Para ese propósito se realizó una propuesta con 50 estudiantes de grado décimo del Colegio Misael Pastrana Borrero de Bogotá D.C., Colombia. Los resultados de la investigación, de tipo cualitativo y de orden descriptivo, indican que, la enseñanza de la Física mediada por simulaciones, laboratorios virtuales y el aprendizaje activo de la física, posibilita motivación, colaboración y altas ganancias en el aprendizaje en los estudiantes participantes del estudio.
\end{abstract}

Palabras clave: tic, educación secundaria, aprendizaje activo de la física, enseñanza de la física

\begin{abstract}
This research analyzes the importance of the use of ICT and the Active Physics Learning strategy in learning of free fall of bodies. For that purpose, a proposal was made with 50 tenth grade students from Misael Pastrana Borrero School in Bogotá, Colombia. The results of the research, qualitative and descriptive, indicate that the teaching of Physics mediated by simulations, virtual laboratories and active learning of physics, promotes motivation, collaboration and high gains in learning in participating students of the study.

Key words: ict, secondary education, active learning of physics, physics teaching
\end{abstract}

\section{Introducción}

Los desarrollos tecnológicos que se han propiciado en la sociedad y han impactado directamente en la vida cotidiana de las personas, no han dejado exento el campo de la educación y la enseñanza de la Física (Velasco \& Buteler, 2017), muestra de ello es la gran variedad de aplicaciones informáticas, simulaciones y laboratorios virtuales, los cuales corresponden a representaciones computacionales de fenómenos naturales que propician el aprendizaje por medio de la interacción (Alessi \& Trollip, 2001). Consolidando así, un campo que se caracteriza

\footnotetext{
${ }^{1}$ Docente-Investigador.Centro de tecnologías para la Academia. Universidad de La Sabana. diego.becerra2@unisabana.edu.co

2 Docente-Investigador.Centro de tecnologías para la Academia. Universidad de La Sabana. ana.vargas@unisabana.edu.co

${ }^{3}$ Docente-Investigador.Centro de tecnologías para la Academia. Universidad de La Sabana. oscar.boude@unisabana.edu.co

${ }^{4}$ Estudiante. Doctorado en Educación-Enfasis en Entornos Virtuales de Aprendizaje. Universidad San Buenaventura.marbemen2015@gmail.com
} 
por plantear nuevas metodologías de enseñanza que buscan articular la informática, la Física y la enseñanza de esta (Bouciguez \& Santos 2010).

El uso de estrategias de Aprendizaje Activo de la Física (AAF) que estructura las actividades de clase en un ciclo o grado con las siguientes etapas: Predicción, Observación, Discusión y Síntesis (PODS), busca mejorar el proceso de enseñanza y la obtención de ganancia en el aprendizaje (Benítez \& Mora, 2013). De igual forma, afirmaciones de Gardner \& Gauld (1990) indican que un factor de motivación para el aprendizaje es el trabajo en el laboratorio, debido a que los estudiantes disfrutan realizar prácticas experimentales.

Asimismo, desde una perspectiva pedagógica Benítez \& Mora (2013) plantean que el AAF es el conjunto de metodologías y estrategias de la enseñanza de la física, en las cuales, los estudiantes son conducidos a construir su propio conocimiento mediante observaciones directas de los fenómenos naturales, para ello, se propone el abordaje de las temáticas de la Física mediante el desarrollo de Clases Demostrativas Interactivas (CID), las cuales plantean el desarrollo de un ciclo de aprendizaje, conocido como el ciclo PODS, de donde se analiza un fenómeno natural y se desarrollan las fases (ver figura 1) de predicción, observación, discusión y síntesis (Sokoloff et. al., 2006).

Figura 1

Ciclo PODS del AAF



Es de resaltar que el Aprendizaje Activo de la Física se puede complementar con el uso de herramientas tecnológicas, como lo plantea Thornton \& Sokolof (1990) estas permiten a los estudiantes:

1) Dirigir y realizar sus prácticas sin invertir tiempo en la recolección de datos para su demostración.

2) Graficar los datos obtenidos en tiempo real, recibir retroalimentación inmediata y analizar los mismos en forma comprensible.

3) Examinar fácilmente las consecuencias de un gran número de cambios en las condiciones experimentales durante una sesión de laboratorio, permitiendo una observación adecuada a la relación de variables de los fenómenos.

Teniendo en cuenta que buena parte de la sociedad actual tiene acceso a la tecnología y elementos como las tablets y/o teléfonos inteligentes, los cuales hacen parte de su vida cotidiana, es común encontrar que la comunicación entre los estudiantes se da por diferentes canales, como el correo electrónico y las redes sociales, también es común encontrar que los estudiantes accedan a diferentes tipos de información por medio de la web, demostrando que los docentes y los libros de texto ya no se conciben como la única fuente de información y posterior conducción al conocimiento. Al respecto, UNESCO (2004) comenta que las TIC son un elemento que 
día a día cobra mayor importancia en el desarrollo del mundo productivo, influyendo en distintos aspectos de él, entro ellos la toma de decisiones, el almacenamiento y la distribución de la información mediante el uso de hardware y software.

Para Cabero (2007) las TIC se han consolidado como un elemento indispensable en los nuevos contextos y escenarios de interacción de los sujetos, y la educación no ha sido ajena a ello, sin embargo, los nuevos escenarios educativos que se configuran, requieren de ejercicios de reflexión que apuesten a la integración crítica de la tecnología, desvirtuando la concepción de ella como un instrumento que automáticamente resuelve las problemáticas educativas.

Algunos autores han indicado que el uso de herramientas como GeoGebra, facilitan la representación conceptual de formas dinámicas que promueven el aprendizaje (Villamizar, 2020). Así como otros establecen, que la integración de este tipo de tecnologías acerca al estudiante a situaciones del mundo real, generando aprendizajes significativos (Gañan, 2020).

En este sentido, Benítez (2015) afirma que las ventajas de usar las simulaciones en el aula de clase son la motivación de los estudiantes gracias a la interactividad, el respeto a los ritmos individuales de aprendizaje, la presentación de imágenes que se recuerdan con más facilidad de forma dinámica y tridimensional de los fenómenos físicos, integrarla teoría y la práctica, aumentar la posibilidad de recordar las variables implícitas en el concepto. Por otra parte, hay estudios que han enfatizado en la eficacia de la gamificación (juegos estructurados) en los procesos formativos de la enseñanza de la física, resaltándose la motivación y la mejora en el desempeño escolar (Batista, Leite \& Braga, 2019).

Las herramientas de hardware y software son generales e independientes de los experimentos, así los estudiantes son capaces de enfocarse en la investigación de muchos fenómenos físicos sin perder tiempo usando instrumentos de uso complicado. El uso de herramientas tecnológicas no determinan ni el fenómeno a investigar, los pasos de la investigación, ni el nivel o sofisticación del objetivo de aprendizaje por lo que son útiles desde el nivel elemental hasta el universitario.

Bajo este panorama, se buscó implementar nuevas estrategias que privilegian el uso de Tecnologías de la Información y la Comunicación y a su vez permitan a los estudiantes alcanzar superar errores conceptuales (Amadeu \& Leal, 2013). Lo anterior tomando en cuenta, que los estudiantes participantes en la investigación presentan fallas conceptuales relacionadas con la adecuada comprensión de conceptos afines con la caída de los cuerpos.

En este sentido, la presente investigación revisa cómo la propuesta del uso del tablero digital, simulaciones, laboratorios virtuales y actividades experimentales combinada con la estrategia de Aprendizaje Activo de la Física, incide en la motivación y el aprendizaje de la caída libre de los cuerposen un grupo de estudiantes de grado décimo del colegio Misael Pastrana Borrero.

\section{Metodología}

La investigación, de carácter cualitativo y de orden descriptivo, mediante la metodología de investigación acción, se desarrolla en seis sesiones de horas cada una, integra la aplicación del uso de tablero digital, simulaciones, laboratorios virtuales, actividades experimentales, con uso de problemáticas de la vida cotidiana y el ciclo que ofrece el Aprendizaje Activo de la Física, buscando que el estudiante potencie el trabajo colaborativo, y mejore el manejo conceptual, al relacionar la teoría y la práctica. 


\subsection{Población y muestra}

La población de la investigación estuvo conformada por estudiantes de grado décimo del Colegio Misael Pastrana Borrero de Bogotá, Colombia, cuyas edades oscilan entre 15 y 17 años. Participaron 27 estudiantes del curso 1001 y 23 estudiantes del curso 10-02 y su estrato socioeconómico oscila entre 1 y 3 . La institución, de carácter público, se encarga del desarrollo de procesos educativos desde el grado preescolar hasta el bachillerato, los rangos de evaluación según el Sistema Escolar de Evaluación (SIE) están distribuidos en una escala numérica de 0 a 5, donde de 0 a 3,0 corresponde a una valoración baja, de 3,0 a 3,9 una valoración básica, de 4,0 a 4,5 una valoración excelente y de 4,6 a 5,0 una valoración superior. Para lograr la participación de la comunidad se contó con el apoyo de la rectora de la institución, quien se encargó de la implementación de los instrumentos y la gestión de los consentimientos informados de los padres de familia.

\subsection{Planteamiento de la estrategia didáctica}

Para el desarrollo de la propuesta, se tuvo presente que de acuerdo con los estándares curriculares de educación para Ciencias Naturales-en el entorno físico, propuestos por el Ministerio de Educación Nacional (MEN), los estudiantes de grado décimo, deben aprender en el componente de mecánica clásica el movimiento de caída libre, razón por la cual la propuesta abordó esta temática. Igualmente, se consideró enmarcar la propuesta como una propuesta didáctica, ya que, de acuerdo con Carrasco (2004), las estrategias didácticas son todas aquellas formas de actuar de un profesor para orientar con pericia el aprendizaje de los estudiantes, concibiendo de esta forma a las estrategias de aula, como todos los actos favorecedores del aprendizaje.

Esta estrategia fusiona en un mismo escenario tres aspectos pedagógicos: el disciplinar, estudaindo un fenómeno físico como lo es la caída libre de los cuerpos; el tecnológico, incorporando un tablero digital, simulaciones, laboratorios virtuales; y el pedagógico, teniendo en cuenta las propuestas del Aprendizaje Activo de la Física. En la investigación, se concibe el rol del docente como mediador en la generación de conocimiento y el desarrollo de las actividades, las cuales están centradas en el estudiante optimizando en ellos los resultados académicos y desarrollo de habilidades sociales. En este sentido, la estrategia contempló tres fases para su desarrollo una fase de preparación, una fase de implementación y una fase de análissi, enmarcadas dentro de una estrategia estructurada en seis momentos (ver figura 2):

Fase de preparación: Inicialmente, se hizo una revisión bibliográfica en la que se observó y clasificó el tema, en esta fase, se aplicó un pretest que permitió la identificación de los errores conceptuales de los estudiantes, igualmente, se verifió el correcto funcionamento de los materiales que se emplearon en la estrategia, tales como el tablero digital y las páginas web donde se pueden encontrar los laboratorios virtuales.

Fase de implementación: Una vez superados los diseños de la estrategia y se comprobó el respectivo funcionamiento de las herramientas se procedió a ejecutarlas en el aula con los estudiantes, en esta fase se toman aspectos relevantes del aprendizaje activo de la física, proponiendo dos situaciones que se trabajan como Clases Interactivas Demostrativas (CID) y el docente desarrolla la clase mediante el ciclo PODS. posteriormente, se realiza la inclusión de las TIC en el aula al utilizar el tablero digital con que cuenta la institución con el fin de que los estudiantes lo manipulen para mejorar la motivación en el aula y al mismo tiempo evidenciar, por medio de un laboratorio virtual, que cuando varios cuerpos con distinta masa que caen desde la misma altura, demoran el mismo tiempo en tocar el piso, luego, se toman aspectos relevantes de la experimentación en la física, en el sentido que se pide a los estudiantes determinar el valor de la aceleración gravitacional en el Colegio Misael Pastrana Borrero y determinar la altura de los tres pisos de la institución sin tener que medirla con una cinta métrica. Finalmente, se retoman las TIC en el aula trabajando con computadoras y diferentes laboratorios virtuales disponibles en la web en los cuales se abordan medidas y características propias de los movimientos Rectilíneo Uniforme, Uniformemente acelerado y caída Libre, en esta parte, los estudiantes trabajan de manera 
colaborativa construyendo gráficas de Velocidad vs Tiempo, Posición vs Tiempo y Aceleración vs Tiempo de cada uno de los movimientos y así determinar si los resultados obtenidos en las gráficas de Caída Libre se asemejan más a un Movimiento Rectilíneo Uniforme o a un Movimiento Uniformemente acelerado.

Fase de análisis: En esta fase configura último momento de la estrategia, en ella se aplicó un pos-test para después de sistematizar los datos determinar si la estrategia de aula fue efectiva o no en cuanto a las ganancias de aprendizaje de los estudiantes del grupo de estudiantes.

Figura 2

Estructura de la estrategia propuesta

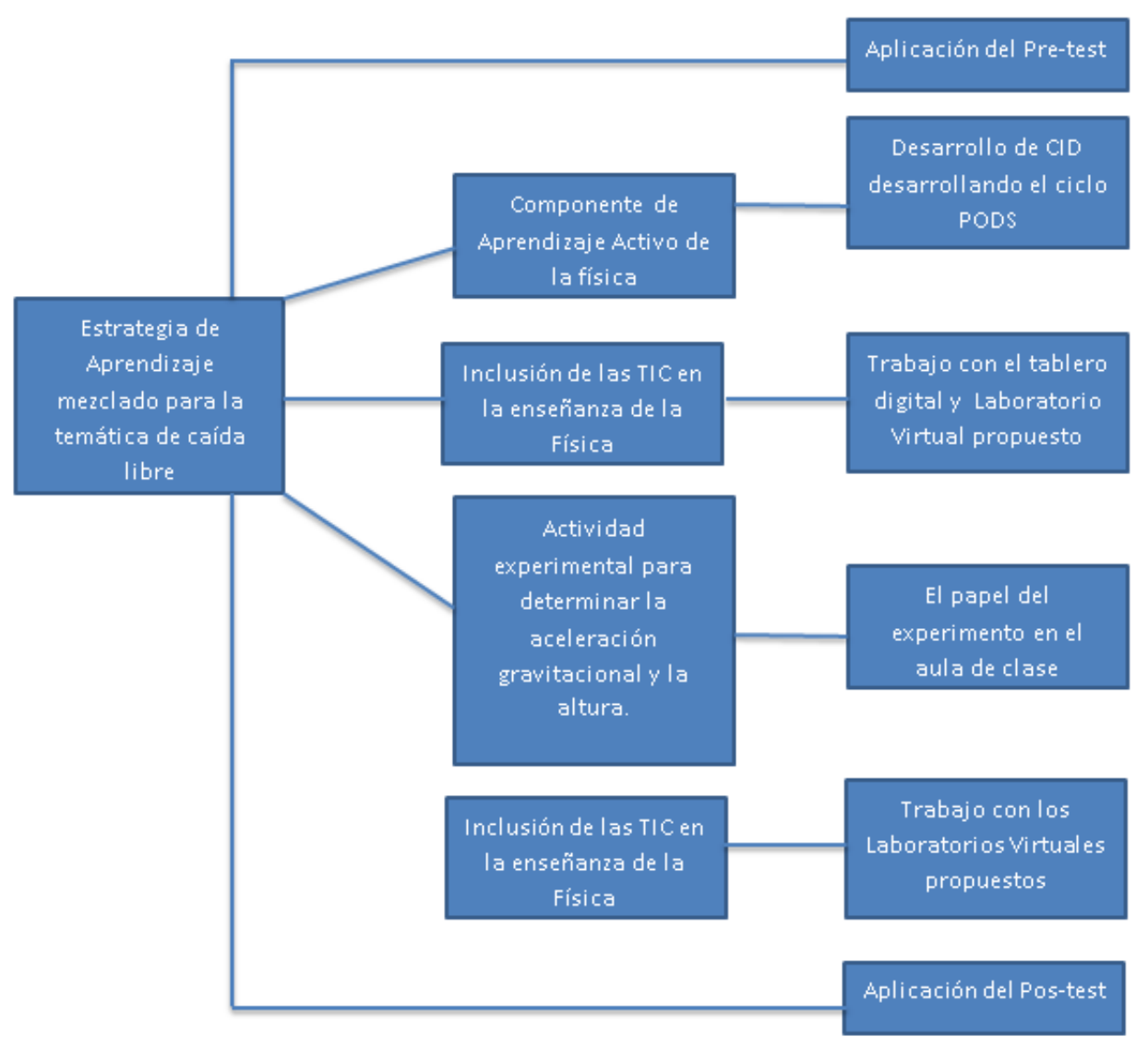

\subsection{Instrumentos}

Tal como lo enuncian Becerra \& Benítez (2019), la aplicación de la prueba spre-test no solo permitió identificar los conocimientos previos de los estudiantes, sino también el diseño de alternativas apropiadas de enseñanza para superar dificultades conceptuales y lograr mejores explicaciones de los fenómenos, a partir del dominio conceptual propio de la física. En este sentido, para evaluar si el trabajo fue efectivo o no, se recolectaron los datos a través de un cuestionario (pre y pos-test) de 5 preguntas de selección múltiple con única respuesta, que fueron tomadas del banco de preguntas de pruebas semestrales con el que cuenta la institución, en los cuales se tomaron en cuenta los conceptos abordados en la estrategia aula y se plantean preguntas relacionadas con conceptos y relaciones de variables de la temática de caída libre, el instrumento no solo contó con la validación de expertos temáticos de la institución, sino que demuestra una confiabilidad obtenida con el alfa de Cronbach de 0,71 , lo cual, según Hernández-Sampieri \& Mendoza (2018) corresponde a una confiabilidad aceptable.

Para evaluar la efectividad de la propuesta, después de aplicar el pre-test antes de la ejecución de la estrategia de aula y el pos-test después de ejecutar los diferentes momentos de la propuesta con los estudiantes, se 
consolidó una matriz de datos del instrumento y se determinó la ganancia de aprendizaje de los estudiantes. Hake (1998) en su trabajo propone una expresión matemática para calcular la ganancia relativa del aprendizaje en estudiantes que presentan evaluaciones de tipo selección múltiple por medio factor g de Hake, de acuerdo a los resultados obtenidos se podrá concluir si efectivamente la estrategia, las herramientas llevadas al aula y su metodología fueron efectivas y cumplieron los objetivos trazados, la expresión matemática es:

$$
g=\frac{\% \text { postest }-\% \text { pretest }}{\text { NotaMáximaPosible }-\% \text { pretest }}(1)
$$

De acuerdo a los resultados obtenidos se establece tres rangos de medida de la ganancia de aprendizaje.

- Zona de ganancia alta sí el factor de Hake cumple con g $\geq 0,7$

- Zona de ganancia media sí el factor de Hake cumple con $0,3 \leq \mathrm{g}<0,7$

- Zona de ganancia baja sí el factor de Hake cumple con $\mathrm{g}<0,3$.

\section{Resultados}

Este apartado presenta los resultados del proceso de investigación a través del desarrollo de cada una de las seissesiones planteadas.

\subsection{Resultados en el desarrollo de la estrategia}

A continuación, se describen los resultados obtenidos en cada una de las sesiones descritas en la metodología.

Aplicación del pre-test: En la sesión se aplicó el pre-test mediante el cual se reconocieron los conocimientos previos y las fallas conceptuales de los estudiantes, entre ellas, se resalta que los estudiantes afirman que los cuerpos más pesados caen a una mayor velocidad que los cuerpos más livianos. Los resultados obtenidos en la sesión mostraron que en promedio el grupo 10-01 obtuvo un promedio de 0,54, mientas el grupo 10-02 obtuvo un promedio de 0,81 , dichos resultados son fundamentales para la obtención de la ganancia de aprendizaje.

CID desarrollando el ciclo PODS: Tal como lo propone el AAF se desarrollaron dos actividades de CID con el fin que los estudiantes desarrollen el ciclo PODS para cada una de ellas y construyan sus conocimientos alrededor de la temática abordada.

- Primera actividad: Se tomó un marcador y una cartuchera que contenía múltiples lápices y marcadores, luego se cuestionó a los estudiantes sobre cuál caería más rápido, si el marcador que tiene una masa determinada o una cartuchera que una cantidad determinada de veces más la misma masa determinada de ese marcador, desarrollando el ciclo PODS. 
Figura 3

Ciclo PODS desarrollado por un grupo de estudiantes

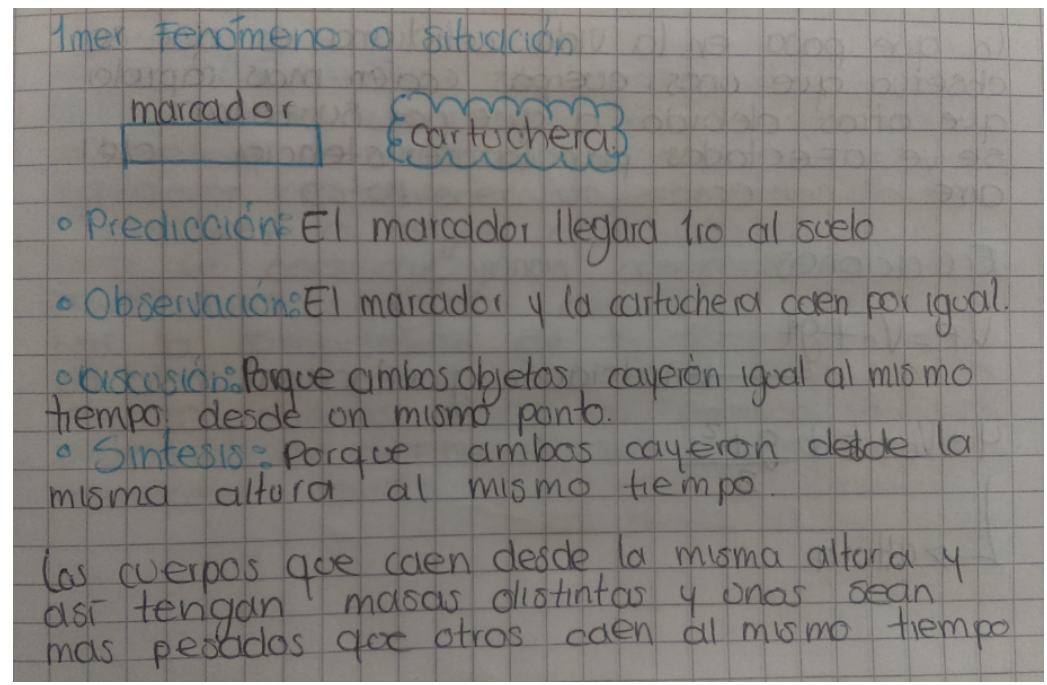

En el inicio de la sesión algunos grupos pensaron que el objeto liviano caería más rápido que el objeto más pesado, y otros pensaron que caería más rápido el objeto más pesado, sin embargo, al final de la actividad los grupos logran comprender que la caída simultánea de dos cuerpos con distinta masa, depende de la aceleración gravitacional con la que caen los objetos a la superficie terrestre y los dos objetos llegan al suelo al mismo tiempo.

- Segunda Actividad: Se tomó una hoja de papel (reciclado) y se dividió exactamente por la mitad, se preguntó a los estudiantes cuál de las dos tendrá más masa o si tienen la misma, luego se arrugó una mitad, pero la otra mitad se dejó intacta y se cuestionó a los estudiantes sobre cuál caería más rápido y porqué, desarrollando el ciclo PODS.

Figura 4

Ciclo PODS desarrollado por un grupo de estudiantes



En esta actividad algunos grupos mencionaron que al tener la misma masa, las dos mitades tebdrían una caída simultanéa, mientras que, a pesar de haber establecido dos mitades exactamente iguales, algunos grupos interpretaron que la mitad arrugada tenía más masa que la mitad extendida. Sin embargo, al final de la actividad los grupos logran comprender que en la vida cotidiana se observa que unos cuerpos caen más rápido que otros, debido a su forma y la resistencia a la caída que proporciona el aire. 
Trabajo con el tablero digital y el laboratorio digital propuesto: Es necesario tener en cuenta que el uso de las TIC no se debe incluir de forma deliberada en los procesos de formación de los estudiantes, sin realizar una planeación previa, de acuerdo con Cabero (2007) se considera necesario hacer un proceso continuo de exploración y reflexión, evaluando en qué medida la inclusión de estas tecnologías propiciarán mejoras en los procesos educativos.

En este sentido, y en aras de propiciar una articulación del aprendizaje activo de la física y las TIC, se desarrolló una CID utilizando el tablero digital que permitió manipular un laboratorio virtual sobre la caída de los cuerpos, el cual se puede encontrar en la dirección web:

http://labovirtual.blogspot.com.co/search/label/Movimiento\%20en\%20la\%20vertical. A partir de esa actividad se preguntó a los estudiantes si todos los cuerpos tardarán el mismo tiempo en tocar el suelo cayendo desde una altura de $55 \mathrm{~m}$ completando el ciclo PODS.

\section{Figura 5}

Entorno del laboratorio virtual sobre caída libre, tomada de Salvador Hurtado Fernández (2013)

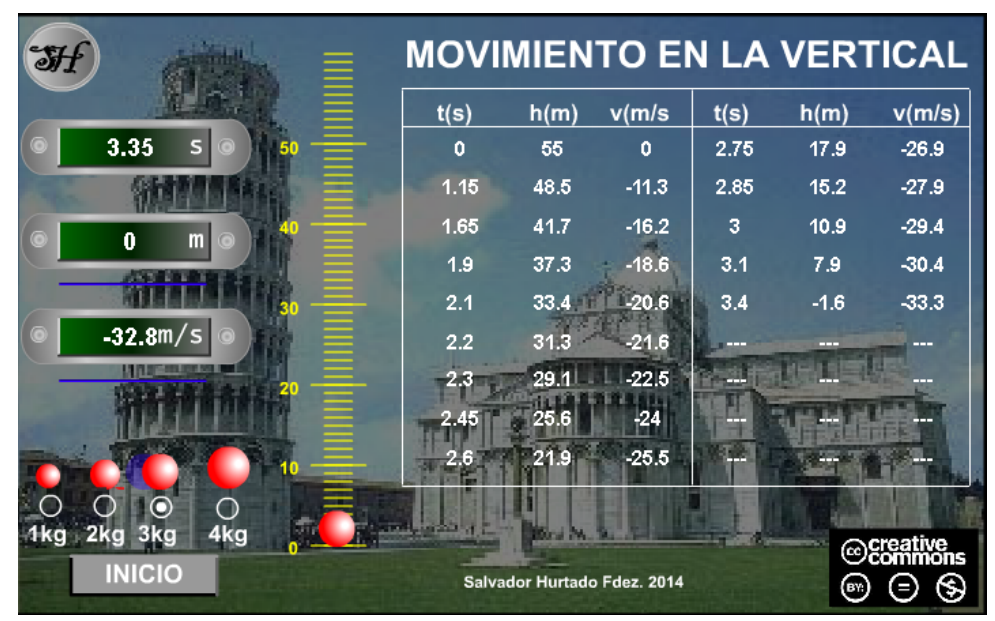

El papel del experimento dentro de la clase: En la ejecución de la propuesta se consideró que la enseñanza de la caída libre de los cuerpos debería ir más allá de aprender de memoria que el valor de la aceleración gravitacional es de $9,8 \frac{\mathrm{m}}{\mathrm{s}^{2}}$ y se propuso a los estudiantes que midieran la altura de la planta física de la institución, luego midieran el tiempo de caída de un objeto y finalmente determinaran el valor de la aceleración gravitacional en el colegio. 


\section{Figura 6}

Actividad experimental

caída libre de los cuerpos

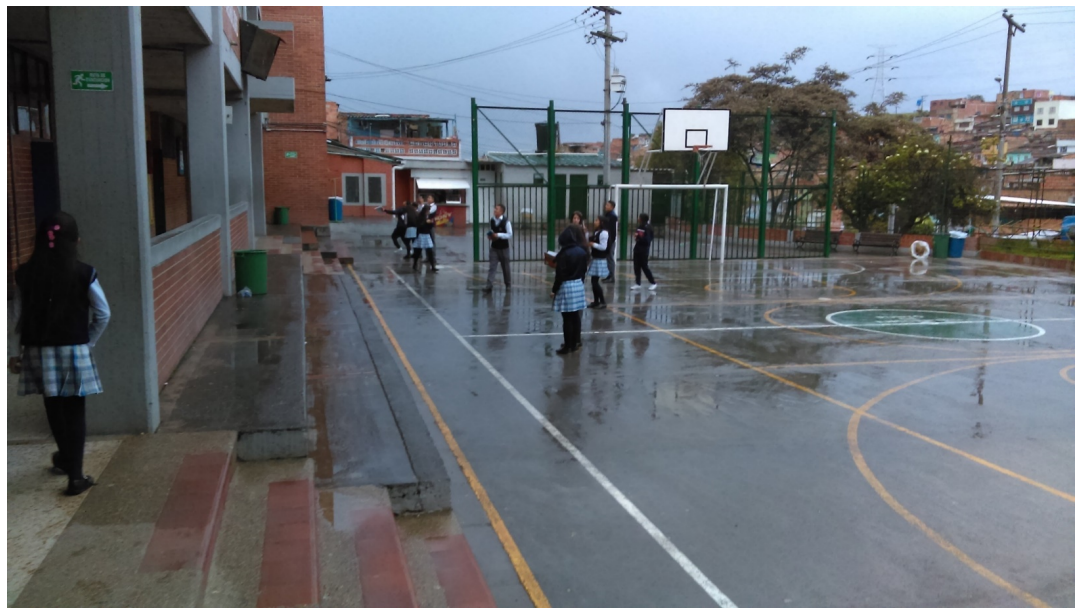

En la realización de la experiencia fue visible expresiones que denotaron la motivación en la realización de la experiencia y el trabajo colaborativo, expresiones como "Me gusta aprender Física fuera del salón de clases" y "Es interesante saber que lo que aprendemos en aula tiene aplicaciones en contextos reales" permiten evidenciar en los estudiantes una actitud favorable para el desarrollo de la asignatura y sus actividades.

Trabajo con los laboratorios virtuales propuestos: En esta sesión se desarrolló un trabajo con los estudiantes y los laboratorios virtuales propuestos sobre el movimiento y caída de los cuerpos, los laboratorios virtuales con los que se trabajó son de acceso libre, fueron desarrollados por Salvador Hurtado Fernández (2013) y están disponibles en el enlace http://labovirtual.blogspot.com.co/p/fisica.html. A diferencia de las sesiones anteriores, en las que se exploraron los laboratorios virtuales con el apoyo del tablero digital, esta sesión se desarrolló en la sala de informática, en ella se observó por medio del enlace:

http://labovirtual.blogspot.com.co/2013/07/movimiento-rectilineo.html como son las características de las gráficas de posición vs tiempo, velocidad vs tiempo y aceleración vs tiempo que se pueden obtener en un movimiento con velocidad constante y un movimiento con aceleración constante, estableciendo sus similitudes y diferencias.

\section{Figura 7}

Entrono de trabajo del laboratorio virtual sobre movimientos, tomada de Salvador Hurtado Fernández (2013)

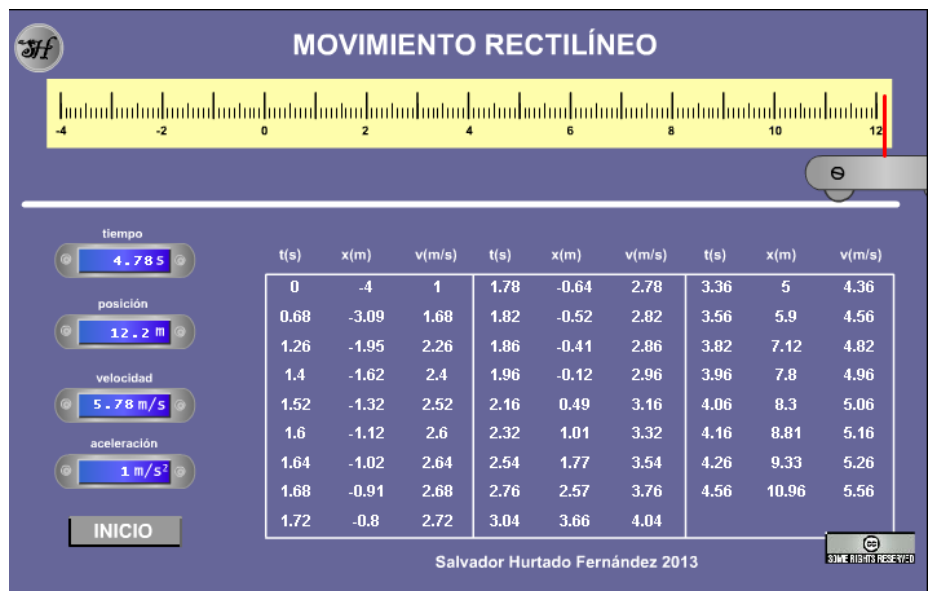


Buscando reforzar lo comprendido en las sesiones anteriores de la investigación, se pidió a los estudiantes que construyeran las gráficas de los movimientos con velocidad y aceleración constante, luego de ello, los estudiantes analizaron las características más similares de un movimiento de caída libre, estableciendo por si mismos que este movimiento atiende a las características de un movimiento con aceleración constante y debido a ello, es que observan la caída simultánea de cuerpos con distinta masa.

Aplicación del pos-test: En la sesión se aplicó el pos-test mediante el cual se reconocen los conocimientos de los estudiantes luego de implementar las actividades, los resultados obtenidos en la sesión mostraron que en promedio el grupo 10-01 obtuvo un promedio de 4,19, mientas el grupo 10-02 obtuvo un promedio de 3,96, dichos resultados son fundamentales para la obtención de la ganancia de aprendizaje.

\subsection{Ganancia de aprendizaje}

Para establecer el nivel de ganancia de aprendizaje y analizar si las actividades propuestas fueron efectivas en cuanto a un nivel de correcta apropiación conceptual por parte de los estudiantes, se utilizó el parámetro de Hake, analizando la cantidad de respuestas acertadas y erróneas de los pre y pos-test en cada grupo.

Tabla 1

Resultados obtenidos en los pre y pos-test

\begin{tabular}{ccc}
\hline Curso & Promedio Pre-test & Promedio Pos-test \\
\hline $10-01$ & 0,54 & 4,19 \\
$10-02$ & 0,81 & 3,96 \\
\hline
\end{tabular}

Determinando las ganancias de aprendizaje de cada curso se obtuvo:

Tabla 2

Ganancias de aprendizaje

\begin{tabular}{|c|c|}
\hline Curso $10-01$ & Curso $10-02$ \\
\hline$g=\frac{4,19-0,54}{5-0,54}=0,81$ & $g=\frac{3,96-0,81}{5-0,81}=0,75$ \\
\hline
\end{tabular}

Analizando los resultados, se observa que los dos grupos obtienen una ganancia significativa de aprendizaje, lo cual permite indicar que para el proceso de enseñanza y aprendizaje la estrategia didáctica planteada facilita a los estudiantes superar los errores conceptuales acerca de la caída libre de los cuerpos.

\section{Discusión}

El estudio mostró que la incorporación del tablero digital, simulaciones, laboratorios virtuales y el Aprendizaje Activo de la Física tiene incidencia significativa en la ganancia de aprendizaje en el caso particular de la caída de los cuerpos, lo cual reafirma resultados anteriores obtenidos en investigaciones que mencionan que la integración de este tipo de tecnologías acerca al estudiante a situaciones del mundo real, generando aprendizajes significativos (Gañan, 2020).

Asimismo, los hallazgos de la investigación coinciden con investigaciones que señalan que el uso de simulaciones y laboratorios virtuales en la enseñanza de la física aumenta la motivación y confianza de los estudiantes para aprender (Becerra, 2014; Rustana, Andriana, Serevina \& junia, 2020). Esto tomando en cuenta que durante la experiencia los estudiantes mantuvieron un rol que les permitió involucrarse en la construcción de los conocimientos, acercados a la realidad. 
En relación a la motivación de los estudiantes no se obtuvieron datos estadísticos que permitan afirmar el incremento en la misma, aunque la valoración se realizó a partir de los comentarios durante y después de la ejecución de la propuesta observando un cambio positivo en la actitud de los estudiantes hacía la clase de física, participando activamente, de forma colaborativa y con gusto, lo cual es coherente con Benítez (2015) que considera que el uso y aplicación de tecnología en el aula genera un mayor acercamiento entre los estudiantes y profesores, minimizando la brecha generacional y haciendo más activa la participación entre los actores del ámbito educativo.

Las TIC en los procesos educativos se reconocen como una necesidad atribuida a los desarrollos tecnológicos de la sociedad González, Bravo \& Ortiz (2018), sin embargo, en dicha incorporación se hace necesaria la exploración, reflexión y evaluación de sus posibles mejoras a los procesos educativos (Cabero, 2007). A partir de los resultados obtenidos en la investigación, se puede establecer que la propuesta de vinculación de las TIC en la enseñanza de la Física es efectiva para consolidar los aprendizajes de los estudiantes, igualmente, los resultados de la investigación son coherentes con las posturas de (Amadeu \& Leal, 2013; Fernández \& Aguirre, 2013; Velasco \& Buteler, 2017; Cherif, Abdelkrim \& Khalid, 2020) al considerar que, con la inclusión de simulaciones y laboratorios virtuales en la enseñanza de la Física, se favorece el desarrollo conceptual, se mejora el proceso de aprendizaje y se potencia el nivel de adquisición de conocimientos de los estudiantes.

De igual forma, con el desarrollo de la investigación, se logra atender a la propuesta de Area (2011) quien considera que en las propuestas de enseñanza, el docente se debe consolidar como un mediador cultural que desarrolla situaciones de enseñanza innovadoras, que van más allá de realizar las mismas prácticas educativas magistrales pero con la vinculación de las TIC, asimismo, se corrobora la propuesta de Bosco (2008) quien resalta que el uso de las TIC en la enseñanza de contenidos, representa un desafío para los docentes quienes tienen la responsabilidad de transformar sus entornos de enseñanza, propiciando la siembra de dudas, e incertidumbres académicas en sus estudiantes, con el fin de conducirlos a una curiosidad intelectual que derive en la construcción de sus conocimientos.

\section{Conclusiones}

Como resultado de la investigación presentada, es posible concluir que existe una relación entre la ganancia de aprendizaje con el uso de simulaciones, laboratorios virtuales y la estrategia del Aprendizaje Activo de la Física, debido a dos factores principales; el primero a la importancia de la planeación y diseño de propuestas de aula que propicien un desarrollo óptimo del proceso de enseñanza y aprendizaje con el uso de las TIC, garantizando la interacción de los estudiantes con las problemáticas de la vida cotidiana en el análisis de los fenómenos físicos.

Así mismo, al revisar los resultados obtenidos para la ganancia de aprendizaje en grupos de grado décimo, se puede afirmar que la propuesta en todo los momentos de ejecución y junto con las herramientas que la componen, mejora la comprensión conceptual de la temática de caída libre y la motivación de los estudiantes favoreciendo el trabajo colaborativo.

Finalmente, se considera que una de las limitantes de la presente investigación estuvo relacionada a la medición de la motivación de los estudiantes con la estrategia, por lo tanto, como prospectiva de la propuesta, se considera interesante analizar si la motivación al usar las TIC es momentánea y al transcurrir el tiempo desaparece, considerando que en el futuro, investigaciones que incluyan este tipo de mediciones, permitíria obtener un análisis con mayor profundidad sobre el desarrollo de este tipo de investigaciones. 


\section{Referencias bibliográficas}

Alessi, S. M. \& R.Trollip, S. (2001). Multimedia for Learning: Methods and Development. Boston: Allyn and Bacon

Amadeu, R. \& Leal, J. (2013). Ventajas del uso de simulaciones por ordenador en el aprendizaje de la Física. Revista Enseñanza de las Ciencias 31 (3), 177-188

Area, M. (2011). Los efectos del modelo 1:1 en el cambio educativo en las escuelas. Evidenciasy desafíos para las políticas iberoamericanas. Revista Iberoamericana de educación, 56, 49-74

Batista, J, Leite, G., \& Braga, J. (2019). Gamification as an active learning strategy in the Physics education. Revista Brasileira de Ensino de Física, 41(4). https://doi.org/10.1590/1806-9126-rbef-20180309

Becerra, D. (2014). Estrategia de aprendizaje basado en problemas para aprender circuitos eléctricos. Revista Innovación Educativa 14 (64) 73-99

Becerra, D. \& Benítez, M. (2019). La Evaluación como una Estrategia de Enseñanza en la Asignatura Electricidad y Magnetismo. Revista Científica, 344-356. Recuperado de: https://revistas.udistrital.edu.co/index.php/revcie/article/view/14505

Benítez, Y. \& Mora, C. (2013). Enseñanza tradicional vs aprendizaje activo para alumnos de ingeniería. Revista Cubana de Física. 27 (2) 175-179

Benítez, M. (2015). La valoración de los applets, por parte del docente, posibilita la uitlización de las TIC en el aula. Revista de investigación \#Hashtag, 105-112.

Bosco, A. (2008). Las tecnologías de la información y la comunicación en la formación del profesorado: lineamientos, actualidad y prospectiva. Razón y Palabra 63. Recuperado dehttp://www.razonypalabra.org.mx/n63/abosco.html

Bouciguez, M. \& Santos, G. (2010). Applets en la enseñanza de la física: un análisis de las características tecnológicas y disciplinares. Revista Eureka Sobre Enseñanza Y Divulgación De Las Ciencias, 7(1), 56-74. Doi: http://dx.doi.org/10.25267/Rev_Eureka_ensen_divulg_cienc.2010.v7.i1.04

Cabero, J. (2007). Las necesidades de las TIC en el ámbito educativo: oportunidades, riesgos y necesidades. Tecnología y comunicación educativas. 21 (45), 4-19. Recuperado de: https://biblat.unam.mx/hevila/Tecnologiaycomunicacioneducativas/2007/vol21/no45/1.pdf

Carrasco, J. (2004). Una didáctica para hoy: Cómo enseñar mejor. España: Ediciones Rialp

Cherif, A. Abdelkrim, E. \& Khalid, E. (2020). Effects of the Integration of PhET Simulations in the Teaching and Learning of the Physical Sciences of Common Core (Morocco). Universal Journal of Educational Research, 8(7), 3014 - 3025. Doi: 10.13189/ujer.2020.080730

Fernández, R. \& Aguirre, C. (2013). ¿Mejoran las simulaciones en los laboratorios de químicael aprendizaje de los alumnos? Percepciones de alumnosuniversitarios de primer curso de Química General. Revista Eureka Sobre Enseñanza Y Divulgación De Las Ciencias, 10 (1), 47-65. Recuperado a partir de https://revistas.uca.es/index.php/eureka/article/view/2793

Gañan, D. (2020). Diseño de un laboratorio virtual para la enseñanza y aprendizaje de la cinemática mediante el uso del software GeoGebra. Números, 104, 147-169. 
Gardner, P. \& Gauld, C. (1990). Labwork and students' attitudes. In E. Hegarty-Hazel (Ed.),The student laboratory and the science curriculum. London: Routledge.

González, A. Bravo, B. \& Ortiz, M. (2018). El aprendizaje basado en simulación y el aporte de las teorías educativas. Revista Espacios, 38 (20). Recuperado de: https://www.revistaespacios.com/a18v39n20/a18v39n20p37.pdf

Hake, R. (1998). Interactive-engagement versus traditional methods: A six-thousand-student survey of mechanics test data for introductory physics courses. American Journal of Physics 66, 64-74

Hernández-Sampieri, R. y Mendoza, C. (2018). Metodología de la Investigación. Ciudad de México: McGraw-Hill Interamericana

Hurtado, S. (2013). Plataforma de laboratorios virtuales Labovirtual. Disponible en: http://labovirtual.blogspot.com/

Rustana, C. Andriana, W. Serevina, V. \& junia, D. (2020). Analysis of student's learning achievement using PhET interactive simulation and laboratory kit of gas kinetic theory. 6th International Conference on Mathematics, Science, and Education (ICMSE 2019) Journal of Physics: Conference Series 1567 (2020) 022011. IOP Publishing. Doi:10.1088/1742-6596/1567/2/022011

Sokoloff, D. et. al. (2006). Active Learning in Optics and Photonics. 1th edition. UNESCO. Paris, France

Thornton, R. \& Sokolof, D. (1990). Learning motion concepts using-time microcomputerbased laboratory tools. American Journal of Physics 58(9), 858-867. Doi: 10.1119/1.16350

UNESCO (2004). Las tecnologías de la información y la comunicación en la formación docente, Guía de planificación. Recuperado de: http://unesdoc.unesco.org/images/0012/001295/129533s.pdf

Velasco, J. \& Buteler, L. (2017). Simulaciones computacionales en la enseñanza de la física: una revisión crítica de los últimos años. Enseñanza de las ciencias, 35 (2), 161-178.

Doi:http://dx.doi.org/10.5565/rev/ensciencias.2117

Villamizar, F. (2020). GeoGebra como herramienta mediadora de un fenómeno físico. Revista do Instituto GeoGebra Internacional de São Paulo, 9(1), 76-89. https://doi.org/10.17227/ted.num45-9832

Esta obra está bajo una Licencia Creative Commons Attribución-NoCommercial 4.0 International

\section{(cc) EY-NC}

